\title{
PRESIÓN TRIBUTARIA EFECTIVA Y POTENCIAL EN ARGENTINA $^{\circ}$
}

\author{
EFFECTIVE AND POTENTIAL TAX EFFORT IN ARGENTINA \\ Marcelo Garriga* \\ Walter Rosales* \\ Norberto Mangiacone*
}

enviado: 10 octubre 2017 - aceptado: 13 marzo 2018

\begin{abstract}
Resumen
El objetivo de este trabajo es estimar la presión tributaria potencial, que permita inferir si la presión tributaria observada en la Argentina está en un sendero sostenible en el tiempo. Se realizan estimaciones econométricas de corte transversal, para una muestra de 178 países con información de un conjunto de variables explicativas de la presión tributaria para el año 2015. Si bien no existen modelos normativos que sugieran un nivel de presión tributaria óptima, hay un amplio tratamiento empírico en la literatura. Los resultados econométricos muestran que en la Argentina la presión tributaria efectiva estaría considerablemente por arriba de su potencial, poniendo en duda la sostenibilidad temporal de la política impositiva.
\end{abstract}

Clasificación JEL: H20, E62.

Palabras clave: presión tributaria, estructura tributaria, política fiscal.

\begin{abstract}
The aim of this paper is to estimate the potential tax effort, which allows to infer if the tax effort observed in Argentina is on a sustainable path. Cross-section

Garriga, M., Rosales, W., \& Mangiacone, N. (2018). Presión tributaria efectiva y potencial en argentina. Estudios económicos, 35 (71), 25-46.

* Centro de Estudios de Finanzas Públicas, FCE - Universidad Nacional de La Plata, Argentina. Correos electrónicos: marcelogarriga@yahoo.com.ar, walter75@yahoo.com, mangiaconenorberto@ gmail.com
\end{abstract}


econometric estimates are made from a sample of 178 countries with data among a set of explanatory variables of tax effort for the year 2015. Although there are no normative models that suggest an optimal level of tax effort, there is a broad empirical treatment in the literature. The econometric results show that in Argentina the effective tax effort would be considerably above the potential tax effort, casting doubt on the temporary sustainability of the tax policy.

JEL Code: H20, E62.

Key Words: Tax Effort, Tax Structure, Fiscal Policy.

\section{INTRODUCCIÓN}

Hace más de 70 años Colin Clark escribía un trabajo en el que señalaba que una presión tributaria mayor al 25 \% del PBI (o 23 \% del Producto Bruto Nacional) provocaría presiones inflacionarias, como resultado del encarecimiento de los bienes de capital. Esto ocurría a la salida de la Segunda Guerra Mundial luego de la cual hubo fuertes presiones para aumentar el gasto público. Clark aclara que esta cifra no surge de un razonamiento estrictamente teórico sino de la evidencia empírica observada en un conjunto de países. Dicho autor envió el trabajo para su publicación a la revista Economic Journal, cuyo editor era J. M. Keynes. En una breve respuesta, Keynes señala: "as a practical proposition I should be strongly disposed to agree... [that] 25 per cent taxation is about the limit of what is easily borne..." (Colin Clark, 1977, p. 23).

Esta discusión trasciende en el tiempo y ha sido motivo de preocupación en ámbitos académicos y de política económica. En particular, en la Argentina ha cobrado fuerza este debate. En la última década, la presión tributaria consolidada en la Argentina (de los tres niveles de gobierno) se incrementó un $70 \%$ pasando de casi el $20 \%$ a algo más del $33 \%$ del PBI. Supera en algunos casos a la de los países desarrollados (Estados Unidos: $26.4 \%$, Corea: $25.3 \%$ ) y a la gran mayoría de los países latinoamericanos (22.8\%). Pareciera existir una inconsistencia entre el nivel de gasto demandado ( $47 \%$ del PBI) y la disposición a pagar los tributos (alta evasión). Esto lleva a que la presión tributaria sobre los que efectivamente pagan sea aún más alta.

Uno de los principales debates que enfrenta una sociedad es el tamaño del sector público. ¿Cuán importante debería ser en relación con el conjunto de la economía? La respuesta de los distintos países en el mundo ha sido muy diferente como muestra la evidencia empírica. Los países pueden tener preferencias por un 
alto nivel de prestación de bienes y servicios públicos (Noruega, Finlandia, Dinamarca, Suecia) pero esto a su vez requiere que los individuos estén dispuestos a tributar los impuestos para financiarlos. No habría una prescripción a priori de cuál es el nivel de gasto público óptimo o deseable en tanto la población esté dispuesta a financiarlo. Los gobiernos, desde distintas visiones políticas, tienen diferentes preferencias por un nivel de gasto público determinado, lo cual requiere recaudar impuestos para sostenerlo.

La pregunta de cuánta recaudación un país puede obtener es muy diferente de cuánto debe recaudar (Langford y Ohlenburg, 2016). La primera puede estar sujeta a investigación empírica, en tanto la segunda depende de cuestiones políticas y técnicas más complejas, tales como las preferencias de la población, los costos de eficiencia y los efectos sobre la equidad de la imposición, la eficiencia con que el sector público gasta los fondos públicos, entre otras.

Como señala Bird (2008), el sistema impositivo de un país es revelador de la estructura económica, la capacidad para administrar impuestos, la demanda por servicios públicos, y el acceso a otras fuentes de recursos como el petróleo. Pero también es determinante la cultura y la moral impositiva, y el nivel de confianza de la población hacia sus gobernantes. La política impositiva no es producto de un gobierno benevolente, sino el resultado de un complejo entramado político y social entre diferentes grupos de intereses, en un determinado contexto institucional y limitado por las capacidades administrativas del gobierno. Cualquier intento de reforma es un ejercicio de legitimación política. Un aspecto central es vincular las decisiones de gasto y recaudación de la manera más transparente posible.

El gran dilema de los países en desarrollo es cómo satisfacer las necesidades de gasto aumentando los impuestos de una manera económicamente eficiente y sostenible, que permita la supervivencia política de los que gobiernan.

En el caso de la Argentina, la comparación de cómo ha evolucionado la presión tributaria (recaudación de impuestos en relación al PBI) con respecto al resto de los países con algunas características similares, y la búsqueda de variables explicativas de este comportamiento, puede ayudar a responder la pregunta de si esta presión impositiva consolidada es sostenible en el tiempo o excede la capacidad contributiva. Dado el desbalance vertical en términos de recaudación de impuestos (provincias y municipios dependen de las transferencias del gobierno nacional), también resulta interesante analizar si es posible realizar reformas a nivel subnacional tendientes a mejorar la performance impositiva sin considerar la presión tributaria agregada. 
Existe una extensa literatura que compara la diferencias en la presión tributaria entre países desarrollados y en desarrollo y al interior de cada grupo. Se han intentado diversas explicaciones para interpretar estas diferencias: PBI per cápita, grado de monetización de la economía, apertura al comercio, naturaleza y composición de los impuestos, grado de corrupción, calidad de la prestación de bienes públicos, grado de urbanización, calidad de las instituciones, preferencias de los consumidores, factores políticos, entre otras.

A efectos de este trabajo, se considera una medida de la presión tributaria definida como los ingresos tributarios ${ }^{1}$ de todos los niveles de gobierno (sector público consolidado), incluyendo recursos provenientes de la seguridad social, como proporción del producto bruto interno de cada país ${ }^{2}$. No se incluye el impuesto inflacionario debido a la falta de información homogénea para el conjunto de países estudiado 3 .

Por su parte, se hace referencia a la presión tributaria potencial como una noción multidimensional, que no se restringe a la capacidad tributaria, sino también a las preferencias de la sociedad por cierto nivel de provisión de bienes públicos (y su financiamiento con impuestos), al nivel socioeconómico de la población, las características demográficas, la distribución del ingreso, la complejidad del sistema tributario, cuestiones políticas y el diseño institucional del país, entre otros factores ${ }^{4}$.

El objetivo de este trabajo es estimar la presión tributaria potencial que permita inferir si la presión tributaria Argentina está en un sendero sostenible en el tiempo. Para este ejercicio se utiliza una muestra de 178 países con información actualizada de un conjunto de variables explicativas de la presión tributaria para el año 2015. A partir de estos datos se realiza un conjunto de estimaciones de corte transversal.

1 En el caso de los municipios se consideran los recursos tributarios propiamente dichos (en los casos de los municipios que cobran impuestos) más los ingresos no tributarios (tasas, derechos y contribuciones). Dada la falta de potestades tributarias, los municipios recurren a tasas con diseños similares a impuestos, no asociados estrictamente a la contraprestación de un servicio (principio del beneficio).

2 Si bien es una medida utilizada en diversos estudios, no está exenta de críticas. En particular, computar los ingresos de la seguridad social generaría heterogeneidades entre países en función del diseño institucional del régimen previsional que se adopte en cada país. Desde otro punto de vista, podría ajustarse el indicador teniendo en cuenta los impuestos negativos de cada país (transferencias de dinero a los ciudadanos).

3 La importancia de considerar el impuesto inflacionario en el indicador de presión tributaria fue observada y sugerida por Juan Llach.

4 Así por ejemplo, puede concebirse un país con alto potencial tributario si tiene un relativamente alto ingreso per cápita, alto nivel educativo de la población y un sistema político transparente y previsible. 
El trabajo se estructura de la siguiente manera: en la sección I se describe la evolución de la estructura impositiva argentina y se la compara con los países de la región y de la OCDE; en la sección II se presentan la metodología y los datos utilizados; en la sección III se muestran los resultados de las regresiones y en la sección IV se analiza para el caso argentino la presión tributaria en relación con su potencial. Finalmente, se presentan las conclusiones.

\section{ESTRUCTURA TRIBUTARIA EN LA ARGENTINA}

La evolución de la presión tributaria en la Argentina se presenta en la tabla 1, comparando los países de la región y de Organización para la Cooperación y el Desarrollo Económicos (OCDE). A lo largo de los 20 años caracterizados en la tabla, se observan algunos hechos estilizados a destacar. En primer lugar, la presión tributaria en la Argentina alcanza un nivel del $33 \%$ en 2015, producto de un notorio crecimiento, acumulando una variación del $77 \%$ en el período analizado (alrededor de 14 puntos del producto). Esto ha colocado al país por sobre el promedio de la región (23\%), solo comparable con Brasil $(32 \%)^{5}$. La comparación con los países de la OCDE muestra a la Argentina ligeramente por debajo del promedio (34 \%), pero superando a países tales como Estados Unidos y Corea del Sur, entre otros. Debe señalarse una característica de varios países de la OCDE, fundamentalmente los europeos, donde el welfare state es muy importante (Pessino y Fenochietto, 2010) a diferencia del caso de Estados Unidos.

Tabla 1. Presión tributaria de países seleccionados.

Expresado en porcentaje del PBI

\begin{tabular}{lcccccc}
\hline PAÍS & 1995 & 2000 & 2005 & 2010 & 2015 & Var. \% ‘95-'15 \\
\hline Argentina & 18.7 & 19.8 & 24.5 & 29.1 & 33.2 & 77.8 \\
Bolivia & 13.8 & 17.6 & 20.0 & 20.3 & 24.7 & 78.1 \\
Brasil & 26.6 & 29.6 & 32.8 & 32.3 & 32.0 & 20.3 \\
Chile & 18.4 & 18.8 & 20.7 & 19.7 & 20.6 & 12.4 \\
Colombia & 15.0 & 14.6 & 18.1 & 18.0 & 20.8 & 38.4 \\
Ecuador & 8.1 & 10.3 & 12.1 & 16.6 & 21.0 & 158.5
\end{tabular}

5 También merece destacarse el importante crecimiento de la presión tributaria en los casos de Bolivia (siendo de los países más pobres de la región) y Ecuador. 


$\begin{array}{lcccccc}\text { Paraguay } & 12.1 & 12.6 & 13.8 & 16.5 & 17.9 & 48.0 \\ \text { Perú } & 15.9 & 14.7 & 16.5 & 17.9 & 17.1 & 7.4 \\ \text { Uruguay } & 21.0 & 21.6 & 23.9 & 26.1 & 27.0 & 28.6 \\ \text { Promedio AL y } & 17.1 & 18.0 & 19.8 & 20.8 & 22.8 & 33.5 \\ \text { Caribe } & 41.9 & 43.1 & 42.8 & 42.0 & 45.5 & 8.6 \\ \text { Francia } & 36.2 & 36.2 & 33.9 & 35.0 & 36.9 & 2.0 \\ \text { Alemania } & 19.1 & 21.5 & 22.5 & 23.4 & 25.3 & 32.1 \\ \text { Corea del Sur } & 40.0 & 41.9 & 42.6 & 41.9 & 38.1 & -4.9 \\ \text { Noruega } & 45.6 & 49.0 & 46.6 & 43.2 & 43.3 & -5.0 \\ \text { Suecia } & 29.8 & 32.8 & 32.5 & 32.5 & 32.5 & 9.0 \\ \text { Reino Unido } & 26.5 & 28.2 & 25.9 & 23.5 & 26.4 & -0.4 \\ \text { Estados Unidos } & 33.3 & 34.0 & 33.6 & 32.6 & 34.3 & 2.9 \\ \text { Promedio OCDE } & \end{array}$

Fuente: elaboración propia sobre la base de OCDE.

El crecimiento de la presión tributaria descrito previamente se asocia además con un cambio de composición de los impuestos que lo integran.

$\mathrm{Al}$ analizar la presión tributaria por nivel de gobierno, se observa que los impuestos nacionales son los que más crecieron ( $74 \%$ acumulado entre 2002 y 2016). En el caso de los impuestos provinciales, el aumento fue del $70 \%$, mientras que el crecimiento a nivel local fue considerablemente más bajo (9\%). Esta dispar evolución ha llevado a una centralización de los recursos en el nivel superior de gobierno.

El mayor crecimiento de la presión tributaria a nivel nacional se explica en los cambios en la composición de su estructura tributaria. Entre los principales factores se encuentran la aparición de nuevos impuestos (débitos y créditos bancarios y retenciones a las exportaciones ${ }^{6}$ ), el aumento de la recaudación de ganancias (producto de la falta de actualización de los mínimos no imponibles en el caso de las personas físicas y del ajuste por inflación en los balances de las empresas) y el incremento de la recaudación de seguridad social (por el cambio de régimen en

6 Las retenciones a las exportaciones tuvieron un peso importante a partir de 2007. En la actualidad, producto de la caída de los precios internacionales y la reducción/eliminación de las alícuotas, la participación relativa de estos recursos viene reduciéndose. 
2008, a partir del cual los aportes personales pasaron a ser parte del financiamiento del sistema público de reparto). Por último, debe destacarse también el fuerte incremento en el IVA explicado básicamente por el aumento de la participación del consumo en el producto.

En el caso provincial, la presión tributaria presenta un crecimiento basado en impuestos sobre el consumo y transacciones. El impuesto a los ingresos brutos muestra un aumento en su importancia relativa dentro de los ingresos tributarios provinciales, a partir de las reformas tributarias implementadas en el 2008 y profundizadas desde 2011. Las modificaciones introducen mayor complejidad en el diseño del impuesto y junto al impuesto de sellos, generan un componente distorsivo sobre la economía. En contraposición, los impuestos patrimoniales vienen perdiendo participación relativa de manera sistemática en la composición tributaria provincial.

Los municipios no presentan cambios significativos en la presión tributaria agregada. Las potestades tributarias limitadas restringen el potencial recaudatorio de los gobiernos locales. A pesar de ello, existen casos donde la estructura tributaria local se torna compleja: impuestos distorsivos disfrazados de tasas (casos de TISH), sobre tasas al impuesto al consumo de combustible y otras tasas con efecto distorsivo (tránsito de mercaderías).

Por último, cabe señalar que este sensible incremento de la presión tributaria se da en un contexto donde se observan altos niveles de evasión. Ello implica que la presión tributaria sobre los que efectivamente pagan los impuestos es todavía más alta. Según estimaciones de Gómez Sabaíni y Morán (2016) la evasión en el IVA alcanzaría el $20 \%$ y en ganancias $50 \%$ tanto en personas físicas como jurídicas ${ }^{7}$. La informalidad en el mercado laboral, que asciende al 34 \% (INDEC, 2016), también es revelador de esta problemática.

\section{CONSIDERACIONES METODOLÓGICAS Y ANÁLISIS DE DATOS}

Se construye una muestra representativa de 178 países, comprendiendo a 29 países latinoamericanos, 35 países pertenecientes al grupo de la OCDE y otros países. Para cada uno de ellos se obtuvo variables explicativas de la presión tributaria conforme a lo utilizado en estudios previos.

7 La tasa de evasión del IVA en los países desarrollados asciende en promedio al 10\%. 
Una descripción de los datos se presenta en la tabla 2, agrupando a los países por nivel de ingreso. Merece destacarse lo siguiente:

- La presión tributaria aumenta con el nivel de ingreso de los países. El promedio de la muestra es de $21.5 \%$. Los países de mayor ingreso tienen una presión tributaria promedio en el orden del $32 \%$, casi el doble que en los países cuyo ingreso per cápita es inferior a U\$S 4000.

- Las economías de los países que muestran una mayor presión tributaria se caracterizan por presentar en general un bajo porcentaje de participación de la agricultura en sus economías, una baja importancia relativa de las rentas originadas en la explotación de recursos naturales, un relativamente alto grado de apertura de la economía, alta formalización, relativamente bajos niveles de corrupción, niveles educativos relativamente altos y bajos niveles de desigualdad.

- Se destaca también consideraciones demográficas. El grupo de países con mayor presión tributaria presenta una tasa de dependencia relativamente alta. Probablemente, una más alta proporción de población envejecida (propia de los países desarrollados) otorga un mayor peso a los sistemas de seguridad social. La tasa de dependencia (mayores de 65 años en proporción al total de población entre 14 y 65 años) está en el orden del $23 \%$, tres veces mayor que el grupo de países de menores ingresos. En un contexto de economía de bienestar, los países recurrirían a mayor presión tributaria para financiar sus mayores gastos. Esta relación es consistente con el resultado encontrado por Mahdavi (2008) y Langford y Ohlenburg (2016), entre otros. Sin embargo, la relación entre presión tributaria y tasa de dependencia presenta en la literatura resultados ambiguos. También podría plantearse que una mayor tasa de dependencia implicaría una menor base imponible (relación negativa con la presión tributaria). Este resultado es encontrado empíricamente, por ejemplo, por Le, Dodson y Bayraktar (2012). 
Tabla 2. Estadísticas descriptivas. Valores medios, 2015

\begin{tabular}{lccccc}
\hline \multicolumn{1}{c}{ Grupo de países } & C1 & C2 & C3 & C4 & C5 \\
\hline $\begin{array}{l}\text { De ingresos per cápita } \\
\text { menores a 4 000 U\$S }\end{array}$ & 99 & 17.5 & 1826.40 & 19.3 & 8 \\
$\begin{array}{l}\text { De ingresos per cápita entre } \\
\text { 4 000 y 20 000 U\$S }\end{array}$ & 46 & 23.1 & 9939.40 & 4.4 & 4.4 \\
$\begin{array}{l}\text { De ingresos per cápita } \\
\text { mayores a 20 000 U\$S }\end{array}$ & 33 & 31.5 & 41156.00 & 1.6 & 2.7 \\
\begin{tabular}{l} 
Total países \\
\hline
\end{tabular} & 178 & 21.5 & 11045.30 & 12.3 & 6.1 \\
\hline
\end{tabular}

Referencias:

C1: Cantidad de países.

C2: Recaudación tributaria (en \% del PBI).

C3: PBI per cápita (U\$S).

C4: Valor agregado de la agricultura en el PBI (en \%).

C5: Rentas de explotación de recursos naturales (en \% del PBI).

Fuente: elaboración propia con base en datos de OCDE, UNDP y Schneider (2016).

Tabla 2 (continuación). Estadísticas descriptivas. Valores medios, 2015

\begin{tabular}{|c|c|c|c|c|c|c|}
\hline Grupo de países & C6 & $\mathrm{C} 7$ & $\mathrm{C} 8$ & C9 & $\mathrm{C} 10$ & $\mathrm{C} 11$ \\
\hline $\begin{array}{l}\text { De ingresos per cápita } \\
\text { menores a } 4000 \text { U\$S }\end{array}$ & 79 & 43.4 & 67.8 & 6.7 & 8.4 & 26.2 \\
\hline $\begin{array}{l}\text { De ingresos per cápita entre } \\
4000 \text { y } 20000 \text { U\$S }\end{array}$ & 98.1 & 31.4 & 53.6 & 9.9 & 15.9 & 25.2 \\
\hline $\begin{array}{l}\text { De ingresos per cápita } \\
\text { mayores a } 20000 \text { U\$S }\end{array}$ & 119.5 & 18.4 & 25.4 & 11.7 & 22.9 & 16.3 \\
\hline Total países & 91.6 & 35.4 & 56.2 & 8.4 & 12.9 & 24.3 \\
\hline $\begin{array}{l}\text { Referencias: } \\
\text { C6: Grado de apertura (mercancía } \\
\text { C7: Índice de economía en negro. } \\
\text { C8: Índice de corrupción. } \\
\text { C9: Años de estudio promedio de } \\
\text { C10: Tasa de dependencia mayore } \\
\text { C11: Índice de desigualdad. }\end{array}$ & $\%$ blación. & & & & & \\
\hline
\end{tabular}




\section{ANÁLISIS ECONOMÉTRICO}

Se emplea el análisis econométrico para obtener estimaciones de la presión tributaria potencial. Se utiliza como variable explicada la presión tributaria observada y como explicativas las variables descriptas en la sección precedente. Se define la presión tributaria potencial de un país como la presión tributaría que podría alcanzar dados los niveles que tomen las variables determinantes a nivel económico, social, político, institucional, demográfico, etcétera.

La estrategia metodológica seguida consiste en testear diferentes modelos con distintas técnicas econométricas (MCO corregido por heterocedasticidad, Tobit y GLM) ${ }^{8}$, las cuales arrojan resultados similares. Se decide tomar los modelos de MCO a los efectos del análisis. Si bien podrían existir variables omitidas (por ejemplo, estructura tributaria, color político de los gobiernos, etc.), este efecto no parece ser condicionante de los resultados. En los test de variables omitidas no se rechaza la hipótesis de independencia entre los residuos y la variable explicada.

Otra posible limitación de estas estimaciones es la endogeneidad. Artana y Templado (2010) advierten que este problema podría presentarse en las variables de economía informal y transparencia (corrupción). Empleando variables instrumentales, los resultados obtenidos no arrojan estimaciones significativas para el instrumento utilizado, sugiriendo que no existe un problema de endogeneidad en el caso estudiado. Por su parte, Gupta (2007) no encuentra que el problema de la endogeneidad en sus estimaciones sea relevante.

La construcción de los distintos modelos se realiza en función de la interpretación conceptual y para evitar los efectos de la multicolinealidad, dada la relativamente alta correlación entre algunas variables. Los resultados se presentan en la tabla 3.

8 La variable endógena está truncada por construcción. En estos casos, el modelo Tobit podría corregir probables sesgos en las estimaciones. Por otro lado, el modelo lineal general (GLM) contempla la posibilidad de varianzas no constantes. Este último modelo fue utilizado por Artana y Templado (2010) para evitar que las estimaciones arrojen valores negativos. 
Tabla 3. Estimaciones econométricas (MCO).

Modelos empíricos para la presión tributaria potencial

\begin{tabular}{|c|c|c|c|}
\hline Variable & 1 & 2 & 3 \\
\hline $\begin{array}{l}\text { Rentas de explotación de recursos } \\
\text { naturales (en \% del PBI) }\end{array}$ & $-0.163^{*}$ & -0.126 & \\
\hline PBI per cápita (U\$S) & $0.000^{*}$ & 0.000 & $0.000 * *$ \\
\hline $\begin{array}{l}\text { Años de estudio promedio } \\
\text { de la población }\end{array}$ & $0.642 * * *$ & $0.469 * *$ & \\
\hline $\begin{array}{l}\text { Dummy países europeos } \\
\text { (igual a 1) }\end{array}$ & $10.495 * * *$ & $10.738^{* * *}$ & $12.753 * * *$ \\
\hline Índice de corrupción & & $-0.188 * * *$ & \\
\hline $\begin{array}{l}\text { Valor agregado de la agricultura } \\
\text { en el PBI (en \%) }\end{array}$ & & & $-0.130 * *$ \\
\hline Constante & $13.644 * * *$ & $26.683 * * *$ & $18.743 * * *$ \\
\hline $\mathrm{R}^{2}$ & 0.514 & 0.60 & 0.483 \\
\hline $\mathrm{N}$ & 174 & 154 & 176 \\
\hline$* \mathrm{p}<.1 ; * * \mathrm{p}<.05 ; * * * \mathrm{p}<.01$ & & & \\
\hline
\end{tabular}

Fuente: elaboración propia

Tabla 3 (continuación). Estimaciones econométricas (MCO). Modelos empíricos para la presión tributaria potencial

\begin{tabular}{|c|c|c|c|c|}
\hline Variable & 4 & 5 & 6 & 7 \\
\hline $\begin{array}{l}\text { Rentas de explotación de } \\
\text { recursos naturales (en \% } \\
\text { del PIB) }\end{array}$ & & & & 0.014 \\
\hline PIB per cápita (U\$S) & & 0.000 & & 0.000 \\
\hline $\begin{array}{l}\text { Años de estudio promedio } \\
\text { de la población }\end{array}$ & & $0.371^{*}$ & & $0.390 *$ \\
\hline $\begin{array}{l}\text { Dummy países europeos } \\
\text { (igual a 1) }\end{array}$ & & $5.651 * * *$ & $6.538 * * *$ & $6.782 * * *$ \\
\hline Índice de corrupción & & & $-0.112 * * *$ & $-0.166 * * *$ \\
\hline
\end{tabular}




\begin{tabular}{lcccc} 
Índice de desigualdad & $-0.206^{* *}$ & & 0.085 \\
$\begin{array}{l}\text { Grado de apertura } \\
\text { (mercancías) en \% }\end{array}$ & $0.055^{* * *}$ & & & 0.014 \\
$\begin{array}{l}\text { Índice de economía } \\
\text { informal }\end{array}$ & $-0.169^{* * *}$ & & & \\
$\begin{array}{l}\text { Tasa de dependencia } \\
\text { mayores 65 }\end{array}$ & & $0.500^{* * *}$ & $0.480^{* * *}$ & $0.252^{* *}$ \\
Constante & $29.337^{* * *}$ & $10.033^{* * *}$ & $19.450^{* * *}$ & $19.647^{* * *}$ \\
$\mathrm{R}^{2}$ & 0.258 & 0.551 & 0.627 & 0.686 \\
$\mathrm{~N}$ & 134 & 175 & 156 & 134 \\
$* \mathrm{p}<.1 ; * * \mathrm{p}<.05 ; * * * \mathrm{p}<.01$ & & & & \\
\hline
\end{tabular}

Fuente: elaboración propia

Los resultados obtenidos son consistentes con lo esperado a priori. El grado de explicación de los distintos modelos $\left(\mathrm{R}^{2}\right)$ oscila entre el $25 \%$ y el $69 \%$. Las variables usualmente analizadas son $\mathrm{PBI}$ pc, que es estadísticamente significativo y positivo en los modelos 1 y 3 , valor agregado de la agricultura presenta resultados similares que recursos naturales (modelo 3 ), grado de apertura de la economía es estadísticamente significativo y positivo (modelo 4 ) e índice de desigualdad presenta un coeficiente estimado negativo y significativo (modelo 4).

En el caso de la renta de recursos naturales, el coeficiente estimado es negativo y significativo (modelo 1). Una interpretación hallada es que la renta de recursos naturales se asocia a una menor presión tributaria del resto de los impuestos. En esta misma línea, los países relativamente dependientes de estas rentas presentarían limitaciones para alcanzar un determinado nivel de presión tributaria (Brum y Diakite, 2016).

Un resultado a destacar es el de la variable dummy de países europeos, cuya estimación de los coeficientes es significativa estadísticamente y positiva. Este resultado podría estar capturando características propias de estos países, asociadas al concepto de welfare state.

Otras variables menos utilizadas en estudios previos arrojan resultados a destacar. El índice de corrupción (que refleja la falta de transparencia) presenta 
coeficientes estimados significativos estadísticamente y negativos (modelos 3, 5 y 7). Este índice representa una forma de medir la calidad institucional, la cual está relacionada con la presión tributaria potencial de un país. Se espera entonces que ante una mayor percepción de corrupción por parte de la comunidad, menor sea la disposición de los contribuyentes a pagar impuestos.

Respecto de la tasa de dependencia de adultos mayores de 65 años, su coeficiente estimado es significativo estadísticamente y positivo (modelos 5, 6 y 7). La influencia de esta variable se origina en la necesidad de financiar mayores gastos asociados a la seguridad social. Desde cierto punto de vista, esta variable no contribuye a ampliar la base imponible (Le, Dodson y Bayraktar, 2012), sino que el vínculo con una mayor presión tributaria potencial está relacionado con la disposición a pagar más impuestos (en línea con los hallazgos de Langford y Ohlenburg, 2016).

El promedio de años de estudios de los ciudadanos muestra en las estimaciones econométricas coeficientes significativos y positivos en los modelos 1, 2, 5 y 7. En la literatura se encuentra una relación positiva entre los niveles educativos de la población y la presión tributaria potencial. Ghura (1998) muestra que la acumulación de capital humano, como una proxy de la provisión de bienes públicos, se asocia a mayor presión tributaria. Este postulado podría generalizarse diciendo que una población más educada es más demandante de bienes públicos y, por lo tanto, tiene una mayor disponibilidad a pagar impuestos. En este sentido, Pessino y Fenochietto (2010) señalan que una población más educada permite entender por qué pagar mayores impuestos.

\section{ANÁLISIS DE LOS RESULTADOS}

Sobre la base de los resultados econométricos, se obtiene estimaciones para cada país de la presión tributaria potencial, dado los niveles observados de las distintas variables explicativas. Resulta útil profundizar en el análisis del caso de estudio. El gráfico 1 muestra la presión tributaria potencial en relación al PBI pc, índice de corrupción, tasa de dependencia ( +65 años) y años promedio de estudio de la población. En todos los casos se destaca la presión tributaria de la Argentina respecto de la recta de ajuste.

Puede observarse el apartamiento de la presión tributaria observada en la Argentina respecto de su potencial. Para un PBI per cápita en torno a U\$S 12500 y el resto de las variables determinantes dadas en su promedio, Argentina tendría una 
presión tributaria potencial del $23 \%$. Sin embargo, la presión tributaria observada se encuentra en $33 \%$ (10 puntos). Análogamente, se obtiene un exceso de presión tributaria respecto de su capacidad considerando el resto de las variables.

Gráfico 1. Presión tributaria potencial y efectiva en Argentina.

Análisis para variables determinantes seleccionadas

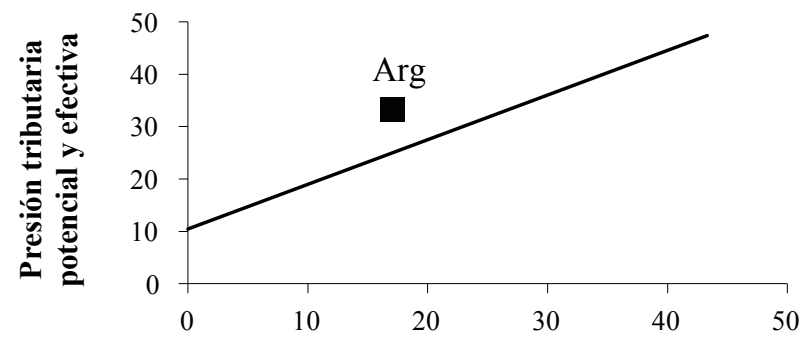

Tasa de dependencia (mayores de 65 años)
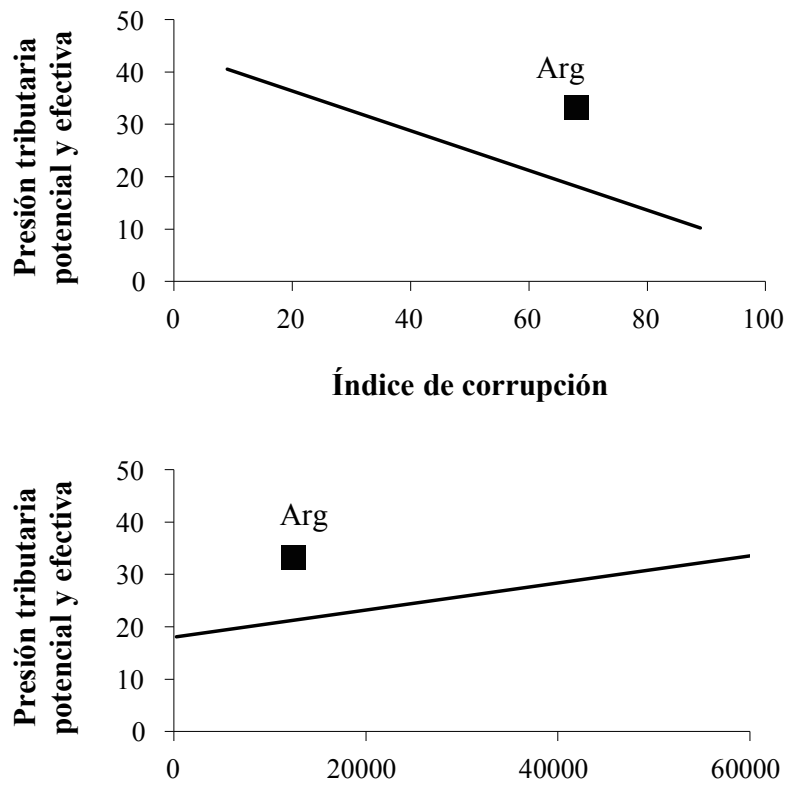

PBI per cápita 


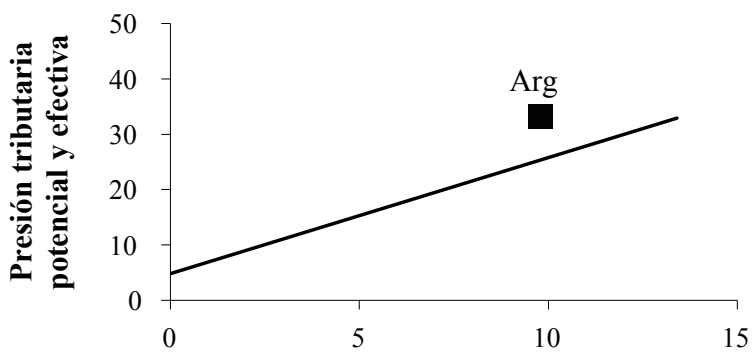

Años promedio de estudio

Fuente: elaboración propia

Siguiendo a Pessino y Fenochietto (2010), se construye el ratio entre presión tributaria observada y potencial estimada para cada país (tabla 4). Puede observarse que la mayoría de los países de la región se encuentran por debajo de su potencial, quedando un margen para aumentar su presión tributaria si lo decidiera así la sociedad. En contraposición, en el caso de la Argentina (al igual que en Bolivia y Brasil), el ratio es muy superior a 1 sugiriendo cierta sobrecarga sobre su sistema tributario.

Tabla 4. Ratio presión tributaria observada/potencial

\begin{tabular}{lc}
\hline País seleccionado & Ratio \\
\hline Argentina & 1.5 \\
Bolivia & 1.2 \\
Brasil & 1.7 \\
Chile & 0.7 \\
Colombia & 0.8 \\
Paraguay & 0.7 \\
Perú & 1.0 \\
Uruguay & 0.9 \\
Venezuela & 0.8 \\
\hline
\end{tabular}

Fuente: elaboración propia.

La sobrecarga tributaria advertida en el caso argentino parece ser un resultado propio de los últimos años. Si bien no existe una medición sistemática del indicador analizado, el relevamiento de distintos estudios, con diversas fuentes de datos y tratamiento metodológico, permite inferir la evolución del caso argentino 
(tabla 5 y gráfico 2). Se destaca como resultado un ratio presión tributaria respecto de su potencial que es creciente en el tiempo. Solo Artana y Templado (2010) dan cuenta de un ratio superior a 1 a partir de 2009.

Tabla 5. Antecedentes de mediciones del ratio presión tributaria/potencial para Argentina en la literatura

\begin{tabular}{|c|c|c|c|}
\hline Autor & Años & $\begin{array}{l}\text { Ratio } \\
\text { estimado }\end{array}$ & Interpretación de los autores \\
\hline $\begin{array}{l}\text { Piancastelli } \\
(2001)\end{array}$ & 1985-1995 & 0.65 & $\begin{array}{l}\text { Factores culturales, institucionales y legales } \\
\text { condicionan la situación fiscal. Aumentar la } \\
\text { presión tributaria por sobre su potencial no } \\
\text { sería la forma de alcanzar el equilibrio fiscal. }\end{array}$ \\
\hline \multirow{2}{*}{$\begin{array}{l}\text { Martín- } \\
\text { Mayoral y } \\
\text { Uribe }(2010)\end{array}$} & 1975 & 0.37 & \multirow{2}{*}{$\begin{array}{l}\text { Los gobiernos latinoamericanos deberían } \\
\text { implementar políticas destinadas a incre- } \\
\text { mentar los ingresos tributarios y así alcan- } \\
\text { zar su potencial. }\end{array}$} \\
\hline & 2006 & 0.45 & \\
\hline Gupta (2007) & 1980-2004 & 0.31 & $\begin{array}{l}\text { La estructura impositiva (directos } v s \text {. indi- } \\
\text { rectos) es importante para acercar la pre- } \\
\text { sión tributaria de los países a su potencial. }\end{array}$ \\
\hline $\begin{array}{l}\text { Pessino y } \\
\text { Fenochietto } \\
(2010)\end{array}$ & 1991-2006 & 0.64 & $\begin{array}{l}\text { El ratio menor a } 1 \text { puede explicarse en in- } \\
\text { eficiencias, o bien en decisiones sociales de } \\
\text { menor recaudación y nivel de gasto. }\end{array}$ \\
\hline $\begin{array}{l}\text { Teera y } \\
\text { Hudson } \\
(2004)\end{array}$ & 2000 & 0.85 & $\begin{array}{l}\text { Un esfuerzo tributario sobre (o bajo) su } \\
\text { potencial puede deberse a la necesidad de } \\
\text { gasto, fuentes de financiamiento, capacidad } \\
\text { administrativas, consideraciones políticas, } \\
\text { entre otras variables. }\end{array}$ \\
\hline $\begin{array}{l}\text { Langford y } \\
\text { Ohlenburg } \\
(2016)\end{array}$ & 1984-2009 & 0.73 & $\begin{array}{l}\text { Los aspectos institucionales son relevantes } \\
\text { en la determinación del potencial tributario. }\end{array}$ \\
\hline $\begin{array}{l}\text { Le, Dodson } \\
\text { y Bayraktar } \\
(2012)\end{array}$ & 1994-2009 & 0.95 & $\begin{array}{l}\text { Mayor calidad institucional (mejor buro- } \\
\text { cracia y menor corrupción) puede aumentar } \\
\text { el potencial tributario. }\end{array}$ \\
\hline
\end{tabular}




\begin{tabular}{|c|c|c|c|}
\hline Autor & Años & $\begin{array}{c}\text { Ratio } \\
\text { estimado }\end{array}$ & Interpretación de los autores \\
\hline \multirow{3}{*}{$\begin{array}{l}\text { Artana y } \\
\text { Templado } \\
(2010)\end{array}$} & \multirow{3}{*}{2009} & \multirow{3}{*}{1.5} & $\begin{array}{l}\text { La presión tributaria en Argentina por so- } \\
\text { bre su potencial es producto de una estruc- } \\
\text { tura tributaria basada en impuestos fáciles } \\
\text { de recaudar (no eficientes). }\end{array}$ \\
\hline & & & $\begin{array}{l}\text { La presión tributaria excesiva redundó en un } \\
\text { aumento del gasto primario, sin impactar en } \\
\text { reducción de la pobreza. }\end{array}$ \\
\hline & & & $\begin{array}{l}\text { El gran salto en el crecimiento de la presión } \\
\text { tributaria no tuvo efectos evidentes en el } \\
\text { crecimiento de la economía. }\end{array}$ \\
\hline
\end{tabular}

Fuente: elaboración propia

Grafico 2. Estimaciones del ratio presión tributaria/potencial para Argentina

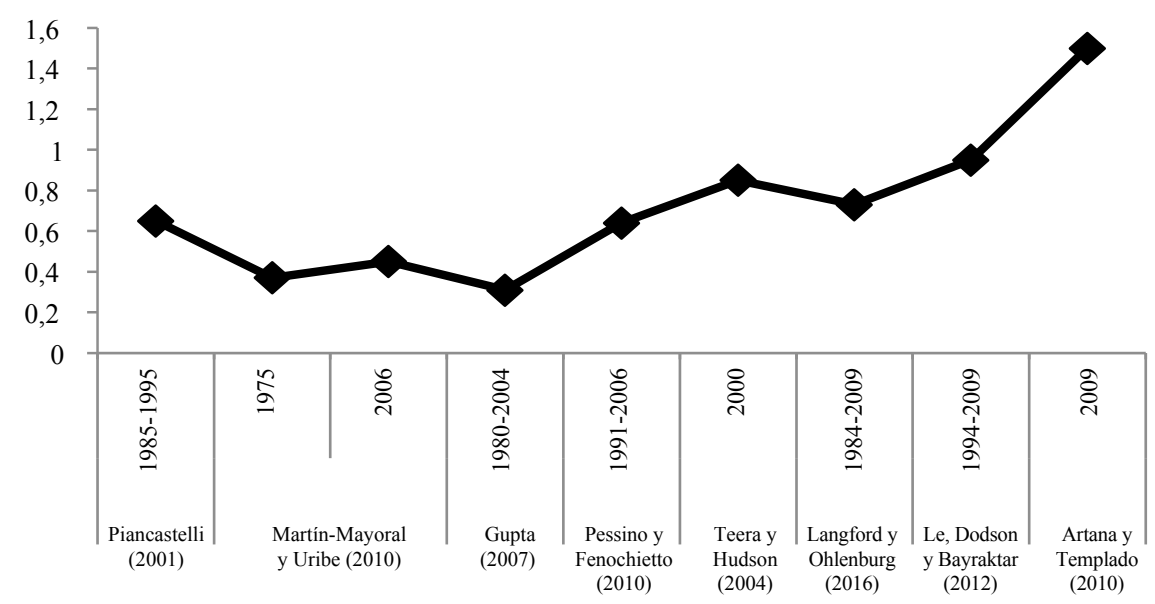

Fuente: elaboración propia en base a trabajos seleccionados 


\section{CONSIDERACIONES FINALES}

El trabajo aborda una problemática de relevancia para la política pública ya que pone bajo análisis el tamaño del sector público y su financiamiento. En particular, este trabajo está enfocado en el caso argentino, en el cual se observa un marcado crecimiento del gasto público en los últimos años, con una presión tributaria sin precedentes históricos.

Normativamente, y como es esperado, no existe un modelo teórico que sugiera un nivel de presión tributaria óptimo. Para abordar el tema en profundidad se distinguen los conceptos de presión tributaria (efectiva u observada) y de presión tributaria potencial. El primer concepto es una medida del peso que tiene la recaudación tributaria en términos del producto. El segundo es un concepto en sentido amplio, ya que no solo refleja aquella presión tributaria posible, que cada país podría alcanzar, sino que también si dicha presión tributaria es aceptable dados ciertos factores condicionantes. De esta forma, un grupo de factores económicos tales como el nivel de actividad en términos per cápita, su composición o el grado de formalización de la economía, entre otros, contribuyen a explicar el tamaño de la base imponible. Por su parte, otro grupo de factores reflejan características sociales, institucionales y demográficas. Por ejemplo, una posibilidad es que las preferencias de la comunidad lleven a aceptar o elegir determinada presión tributaria. Así puede ocurrir que una economía sea relativamente rica, pero que la sociedad prefiera un menor peso relativo del sector público y, por lo tanto, no esté dispuesta a aceptar una alta tributación. Distinto es el caso de países cuyo path dependency está asociado al concepto del welfare state, en los cuales la población demanda al Estado la provisión de una amplia gama de bienes públicos. En estos casos, la disposición a pagar impuestos es relativamente más alta, y en línea con ello, mayor sería la presión tributaria potencial.

Para estimar la presión tributaria potencial de cada país, se construye una muestra de 178 países que incluye una medida de la presión tributaria observada en cada uno de ellos. Como variables explicativas se consideran las variables generalmente utilizadas en la literatura, tales como PBI per cápita, participación de la agricultura en la economía, grado de apertura, renta de explotación de recursos naturales y desigualdad del ingreso. Pero también se incluyen variables menos exploradas, entre las que se encuentran el índice de corrupción, la tasa de dependencia del grupo de población mayor a 65 años y los años promedio de estudio. En general, los estudios empíricos explican una parte de la presión tributaria. Queda por explorar un componente importante de la misma. Las estimaciones econométri- 
cas arrojan resultados consistentes con lo esperado. Las predicciones de los modelos testeados corresponden a una estimación de la presión tributaria potencial. Este resultado es utilizado para contrastar la presión tributaria en el caso de la Argentina. Se observa que la presión tributaria se encuentra muy por arriba de su potencial.

Varias implicancias e interrogantes de desprenden de los resultados obtenidos para el caso argentino.

¿Cuán sostenible podría ser mantener niveles altos de presión tributaria? El ratio presión tributaria/presión tributaria potencial muestra a la Argentina en niveles en torno al 1.5. Este ratio es aún más relevante teniendo en cuenta que la evasión tributaria es muy elevada. Es decir, la presión tributaria sobre los que efectivamente pagan los impuestos es todavía más alta.

¿Cómo se llegó a alcanzar tal presión tributaria, muy por arriba de su potencial? Es necesario indagar más allá del enfoque empírico empleado. Algunas consideraciones de economía positiva podrían aportar explicaciones. Una de ellas es la temporalidad de los hechos. El Estado aprovechó la oportunidad de un ciclo expansivo de la economía (con altos precios de las materias primas exportables) para aumentar la presión tributaria en impuestos distorsivos, poco visibles, que capturan rentas temporarias (entre otras, impuesto al cheque, retenciones a las exportaciones, estatización del sistema previsional). Otras consideraciones vienen por el lado de la ilusión fiscal, dado que aumentó la importancia de determinados impuestos de amplia performance recaudatoria que son menos visibles para la comunidad (por ejemplo, ingresos brutos).

Este panorama de alta presión tributaria (33 \% del PBI) se agrava en un contexto en el que el producto per cápita no crece desde el año 2011 (incluso cae en algunos años), el gasto público consolidado asciende en 2015 al $47 \%$ del PBI y el déficit fiscal supera el $7 \%$ del PBI. ¿Cuán sustentable podría ser mantener este alto nivel de gasto público, si la presión tributaria está considerablemente alejada de su potencial? Si bien la respuesta es compleja, un aspecto para el análisis son las características del sistema tributario. Mirrless (2011) identifica que en un buen sistema tributario interviene el concepto de justicia, fairness, noción que va más allá de las consideraciones distributivas. Este concepto está vinculado con la aceptación del impuesto por parte de la comunidad. La alta presión tributaria combinada con elevados niveles de evasión le quita legitimidad y sentido de justicia al sistema impositivo. De igual forma, la baja calidad de los bienes y servicios públicos provistos por 
el Estado en la Argentina (salud, educación, seguridad, entre otros) también hace que la estructura tributaria sea deslegitimada por la sociedad.

Más allá de lo señalado, existen dimensiones estructurales que contribuyen a ampliar los límites sobre los cuales se puede extender la presión tributaria, pero que exceden los parámetros que se abordan típicamente en una reforma tributaria. Una mejora en la transparencia (menor percepción de corrupción) y en la prestación de bienes públicos podría contribuir a una mayor disposición a pagar impuestos. Varios de estos aspectos suponen cambios en el largo plazo (instituciones democráticas, nivel educativo de la población, estabilidad política, etc.). Estos aspectos llevan a lograr la legitimidad de la comunidad por el sistema tributario.

\section{REFERENCIAS BIBLIOGRÁFICAS}

Artana, D., \& Templado, I. (2010). Is the Argentine Revenue Effort too high? FIEL, Documento de Trabajo n. ${ }^{\circ} 106$.

Bahl, R. (1971). A Regression Approach to Tax Effort and Tax Ratio Analysis. IMF Economic Review, 18(3), 570-612.

Bird, R., Martinez-Vazquez, J., \& Torgler, B. (2008). Tax Effort in Developing Countries and High Income Countries: The Impact of Corruption, Voice and Accountability. Economic Analysis \& Policy, 38(1), 55-72.

Bird, R., Martinez-Vazquez, J., \& Torgler, B. (2014). Societal Institutions and Tax Effort in Developing Countries. Annals of Economics and Finance, Society for AEF, 15(1), 301-351.

Bornhorst, F., Gupta, S., \& Thornton, J. (2009). Natural resource endowments and the domestic revenue effort. European Journal of Political Economy, 25(4), 439-446.

Brun J., \& Diakité M. (2016). Tax Potential and Tax Effort: An Empirical Estimation for Non-resource Tax Revenue and VAT's Revenue. CERDI, Working Paper, n. ${ }^{\circ} 10$.

Buehn, A., \& Schneider, F. (2016). Estimating the Size of the Shadow Economy: Methods, Problems and Open Questions. IZA, Discussion Paper Series n. ${ }^{\circ}$ 9820.

Chand, S., \& Moene, K. (1997). Controlling Fiscal Corruption. IMF, Working Paper n. ${ }^{\circ} 97 / 100$.

Chelliah, R. (1971). Trends in taxation in developing countries. IMF Economic Review, 18(2), 254-331.

Chelliah, R., Baas, H., \& Kelly, M. (1975). Tax ratios and tax effort in developing 
countries, 1969-71. IMF Economic Review, 22(1), 187-205.

Clark, C. (1977). The scope for, and limits of, taxation. En The State of Taxation (pp. 19-28). London: Institute of Economic Affairs.

Clist, P., \& Morrissey, O. (2011). Aid and Tax Revenue: Signs of a Positive Effect Since the 1980s. Journal of International Development, 23(2), 165-180.

Ghani, Z. (2012). A cross country analysis of tax performance with special focus on Pakistan's tax effort (Proyecto de Maestría). Swedish Business School at Orebro University, Suecia.

Ghura, D. (1998). Tax Revenue in Sub-Saharan Africa: Effects of Economic Policies and Corruption. IMF, Working Paper n. ${ }^{\circ}$ 98/135.

Gomez Sabaini, J. \& Morán D. (2016). Evasión tributaria en América Latina. Nuevos y antiguos desafíos en la cuantificación del fenómeno en los países de la región. Comisión Económica para América Latina y el Caribe, Serie Macroeconomía del Desarrollo n. ${ }^{\circ} 172$.

Gupta, A. (2007). Determinants of Tax Revenue Efforts in Developing Countries. IMF, Working Paper n. ${ }^{\circ} 184$.

Hassan, M., \& F. Schneider (2016). Size and Development of the Sadow Economies of 157 Countries Worldwide: Updated and New Measures from 1999 to 2013. IZA, Discussion Papers n. ${ }^{\circ} 10281$.

Karagöz, K. (2013). Determinants of tax revenue: does sectorial composition matter? Journal of Finance, Accounting and Management, 4(2), 50-63.

Keen, M., \& Lockwood, B. (2006). Is the VAT a Money Machine? National Tax Journal, 59(4), 905-928.

Kimakova, A. (2009). Government size and openness revisited: the case of financial globalization. Kyklos, 62(3), 394-406.

Langford, B., \& Ohlenburg, T. (2016). Tax Revenue Potential and Effort, an empirical investigation. IGC, Working Paper.

Le, T., Bayraktar, N., \& Moreno-Dodson, B. (2012). Tax Capacity and Tax Effort: Extended Cross-Country Analysis from 1994 to 2009. Policy Research, Working Paper.$^{\circ}$ 6252. World Bank.

Lotz, J., \& Morrs, E. (1967). Measuring 'Tax Effort' in Developing Countries. IMF Economic Review, 14(3), 478499.

Mahdavi, S. (2008). The level and composition of tax revenue in developing countries: Evidence from unbalanced panel data. International Review of Economics \& Finance, 17(4), 607-617.

Martín-Mayoral, F. \& Uribe, C. (2010). Determinantes económicos e institucionales del esfuerzo fiscal en América Latina. Investigación Económica, 69(273), 85-113.

Mirrlees, J., Adam, S., Besley, T., Blundell, R., Bond, S., Chote, R., Gammie, M., Johnson, P., Myles, G. \& Poterba, J. (2011). Tax by Design. The Mirrlees 
Review. Oxford: Oxford University Press.

Pessino, C., \& Fenochietto, R. (2010). Determining countries' tax effort. Hacienda Pública Española, IEF, 195(4), 65-87.

Piancastelli, M. (2001). Measuring the Tax Effort of Developed and Developing Countries: Cross Country Panel Data Analysis - 1985/95. IPEA, Discussion Paper n. ${ }^{\circ} 818$.

Puviani, A. (1897). Teoría della illusione nelle entrate pubbliche. Perugia: Unione Tipografica Cooperativa.

Rodrik, D. (1998). Trade Policy and Economic Performance in Sub-Saharan Africa. NBER, Working Paper n. ${ }^{\circ} 6562$.

Tait, A., Gratz, W., \& Eichengreen, B. (1979). International comparisons of taxation for selected developing countries 1972-76. IMF Economic Review, 26(1), 123156.

Tanzi, V., \& Davoodi, H. (2001). Corruption, Growth and Public Finances. Arvind K. Jain Editorial, Political Economy of Corruption (pp.89-110), London: Routledge.

Teera, J., \& Hudson, J. (2004). Tax Performance: A Comparative Study. Journal of International Development, 16(6), 785-802.

Vegh, C., Gunter, S., Riera-Crichton, D., \& Vuletin, G. (2017). Non-linear distortionbased effects of tax changes on output: a worldwide narrative approach. IDB, Discussion Paper n. 540.

Wahid, A. (2008). An empirical investigation on the nexus between tax revenue and government spending: The case of Turkey. International Research Journal of Finance and Economics, 16, 46-51.

(C) 2018 por los autores; licencia otorgada a la revista Estudios económicos. Este artículo es de acceso abierto y distribuido bajo los términos y condiciones de una licencia Atribución-No Comercial 3.0 Unported (CC BY-NC 3.0) de Creative Commons. Para ver una copia de esta licencia, visite http://creativecommons.org/ licenses/by-nc/3 